\title{
An efficient way to model complex magnetite: Assessment of SCC-DFTB against DFT
}

Cite as: J. Chem. Phys. 150, 094703 (2019); https://doi.org/10.1063/1.5085190

Submitted: 10 December 2018 . Accepted: 12 February 2019 . Published Online: 01 March 2019

Hongsheng Liu (D), Gotthard Seifert, and Cristiana Di Valentin (D)
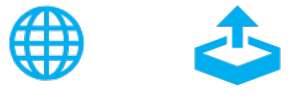

\section{ARTICLES YOU MAY BE INTERESTED IN}

Modelling realistic $\mathrm{TiO}_{2}$ nanospheres: A benchmark study of SCC-DFTB against hybrid DFT The Journal of Chemical Physics 147, 164701 (2017); https://doi.org/10.1063/1.4994165

Nonlinear polarization evolution using time-dependent density functional theory The Journal of Chemical Physics 150, 094101 (2019); https://doi.org/10.1063/1.5068711

Nonlinear-response properties in a simplified time-dependent density functional theory (sTD-DFT) framework: Evaluation of excited-state absorption spectra

The Journal of Chemical Physics 150, 094112 (2019); https://doi.org/10.1063/1.5080199

The Journal

of Chemical Physics

The Emerging Investigators Special Collection and Awards Recognizing the excellent work of early career researchers! 


\title{
An efficient way to model complex magnetite: Assessment of SCC-DFTB against DFT
}

\author{
Cite as: J. Chem. Phys. 150, 094703 (2019); doi: 10.1063/1.5085190 \\ Submitted: 10 December 2018 - Accepted: 12 February 2019 • \\ Published Online: 1 March 2019
}

Hongsheng Liu, ${ }^{\text {(D) Gotthard Seifert, }{ }^{2} \text { and Cristiana Di Valentin }}{ }^{1}$

\begin{abstract}
AFFILIATIONS
${ }^{1}$ Dipartimento di Scienza dei Materiali, Università di Milano-Bicocca, Via R. Cozzi 55, I-20125 Milano, Italy

${ }^{2}$ Theoretische Chemie, Technische Universität Dresden, 01062 Dresden, Germany
\end{abstract}

a) Author to whom correspondence should be addressed: cristiana.divalentin@unimib.it

\begin{abstract}
Magnetite has attracted increasing attention in recent years due to its promising and diverse applications in biomedicine. Theoretical modelling can play an important role in understanding magnetite-based nanomaterials at the atomic scale for a deeper insight into the experimental observations. However, calculations based on density functional theory (DFT) are too costly for realistically large models of magnetite nanoparticles. Classical force field methods are very fast but lack of precision and of the description of electronic effects. Therefore, a cheap and efficient quantum mechanical simulation method with comparable accuracy to DFT is highly desired. Here, a less computationally demanding DFT-based method, i.e., self-consistent charge density functional tight-binding (SCC-DFTB), is adopted to investigate magnetite bulk and low-index (001) surfaces with newly proposed parameters for $\mathrm{Fe}-\mathrm{O}$ interactions. We report that SCC-DFTB with on-site Coulomb correction provides results in quantitatively comparable agreement with those obtained by DFT $+\mathrm{U}$ and hybrid functional methods. Therefore, SCC-DFTB is valued as an efficient and reliable method for the description magnetite. This assessment will promote SCC-DFTB computational studies on magnetite-based nanostructures that attract increasing attention for medical applications.
\end{abstract}

Published under license by AIP Publishing. https://doi.org/1 0.1063/1.5085190

\section{INTRODUCTION}

Magnetite $\left(\mathrm{Fe}_{3} \mathrm{O}_{4}\right)$, a ferrimagnet with a high Curie temperature of about $850 \mathrm{~K}$, has attracted increasing attention in recent years due to its promising applications in biomedicine, including drug delivery, magnetic resonance imaging (MRI), magnetic hyperthermia, bioseparation, and biosensors. ${ }^{1-7}$ At room temperature, magnetite crystallizes in an inverse spinel structure with oxygen anions arranged in a slightly distorted face centered cubic lattice and iron atoms occupying tetrahedral and octahedral interstitial sites. Both $\mathrm{Fe}^{2+}$ and $\mathrm{Fe}^{3+}$ exist in magnetite in a ratio of $1: 2$, with tetrahedral sites occupied by $\mathrm{Fe}^{3+}$ and octahedral sites occupied by an equal number of $\mathrm{Fe}^{2+}$ and $\mathrm{Fe}^{3+} .8$

In general, the applications of magnetite in biomedicine are based on the attachment of ligands to $\mathrm{Fe}_{3} \mathrm{O}_{4}$ nanoparticles, which is clearly a surface issue. Therefore, a lot of effort has been devoted to the investigation of magnetite surfaces, as summarized in a recent review. ${ }^{9}$ For the $\mathrm{Fe}_{3} \mathrm{O}_{4}(001)$ surface, considering the $(\sqrt{ } 2 \times \sqrt{ } 2) \mathrm{R} 45^{\circ}$ reconstruction that appears in experiments, ${ }^{10-15}$ Pentcheva and co-workers proposed a distorted bulk truncation
(DBT) model based on density functional theory (DFT) calculations, ${ }^{16}$ which is thermodynamically very stable. ${ }^{16,17}$ However, the Pendry reliability factor $\left(R_{P}\right)$ for the low-energy electron diffraction (LEED) investigation was somewhat poor $\left(R_{P}=0.34\right),{ }^{18}$ and the DBT model could not explain the site preference of $\mathrm{Au}$ adatoms deposited on the $\mathrm{Fe}_{3} \mathrm{O}_{4}(001)$ surface. ${ }^{19}$ In 2014, Bliem et al. proposed a subsurface cation vacancy (SCV) model, that is a B layer terminated $\mathrm{Fe}_{3} \mathrm{O}_{4}(001)$ surface, with an extra interstitial $\mathrm{Fe}_{\mathrm{Tet}}$ atom in the second layer replacing two $\mathrm{Fe}_{\mathrm{Oct}}$ atoms that are removed from the third layer per $(\sqrt{ } 2 \times \sqrt{ } 2) \mathrm{R} 45^{\circ}$ unitcell. ${ }^{20}$ The SCV model agrees well with the surface X-ray diffraction ${ }^{21}$ and shows a much better agreement with experimental LEED IV $\left(\mathrm{R}_{\mathrm{P}}=0.125\right)^{20}$ compared with the DBT model $\left(\mathrm{R}_{\mathrm{P}}=0.34\right) .{ }^{18}$ In addition, the SCV model shows, at the DFT + U level of theory, a higher thermodynamic stability than DBT over the entire range of oxygen chemical potentials accessible in experimentally conditions and can well explain the site preference of $\mathrm{Au}$ adatoms when deposited on the $\mathrm{Fe}_{3} \mathrm{O}_{4}(001)$ surface. ${ }^{20}$ In contrast, the structure of the $\mathrm{Fe}_{3} \mathrm{O}_{4}(111)$ surface is not yet clear because there are two competitive terminations that are close in energy at oxygen poor conditions and are difficult to distinguish 
by STM. ${ }^{9}$ The $\mathrm{Fe}_{3} \mathrm{O}_{4}(110)$ surface is also not well understood up to now, though several experimental ${ }^{22-24}$ and computational ${ }^{25-27}$ studies have been performed. Besides the termination of surfaces, molecular adsorption, such as that of water, ${ }^{28-36}$ carbon monoxide, ${ }^{37}$ formic acid, ${ }^{38}$ methanol, ${ }^{39}$ polyvinyl alcohol, ${ }^{36}$ polyethylene glycol, ${ }^{36}$ and so on, has also been widely explored.

However, in contrast to the large amount of fundamental studies on magnetite bulk and surfaces, magnetite nanoparticles, which are the mostly interesting structures in nanomedicine, have only been studied experimentally and in reference to their synthesis and medical applications. ${ }^{1-7}$ Only few computational studies exist on the organic molecule adsorption on magnetite nanoparticles, based on force-field methods, ${ }^{40-43}$ which intrinsically lack the description of electronic and magnetic effects and of the chemical processes at the interface. First-principles calculations may play an important role in the achievement of an atomic level understanding of magnetite nanoparticle structure and properties. However, standard DFT methods fail in the detailed description of electronic and magnetic properties of magnetite. ${ }^{44}$ More sophisticated approaches are required to catch proper effects, such as DFT $+\mathrm{U}$ or hybrid functionals, which, however, are very expensive and time consuming. Therefore, the scientific community is in urgent need for a cheaper method but still sufficiently accurate for such a complex material as magnetite.

The self-consistent charge density functional tight binding $(\mathrm{SCC}-\mathrm{DFTB})^{45}$ is an approximate quantum chemical method derived from DFT based on a second-order expansion of the DFT total energy expression. SCC-DFTB, which is a fast and efficient quantum mechanical simulation method, provides information about electronic structure properties, which is not available from classical force fields. In the past few years, SCC-DFTB has been successfully exploited to investigate various types of systems, including semiconductor $\left(\mathrm{Si} / \mathrm{SiO}_{2}\right.$ interfaces $\left.{ }^{46}\right)$, hybrid inorganicorganic systems (gold-thiolate compounds ${ }^{47}$ and organic molecules on the $\mathrm{GaAs}(100)$ surface $\left.{ }^{48}\right)$, low dimensional materials $\left(\mathrm{MoS}_{2}\right.$ nanotubes ${ }^{49}$ chrysotile nanotubes, ${ }^{50}$ and $\mathrm{MoS}_{2}$ fullerenes ${ }^{51}$ ), transition metal and relative metalorganic complexes, ${ }^{52}$ and even transition metal oxides ( $\mathrm{TiO}_{2}$ bulk, surfaces, and nanoparticles). ${ }^{53-61}$

In this study, we extend the available DFTB parametrizations for the description of magnetite, proposing a parametrization of the $\mathrm{Fe}-\mathrm{O}$ interactions. The performance of these new parameters is assessed through the comparison of SCC-DFTB data for several structural, electronic, and magnetic properties of magnetite bulk and (001) surfaces with those obtained by DFT $+\mathrm{U}$ and hybrid functional calculations in previous works. ${ }^{16,20,34,44}$ Our study shows that results by SCC-DFTB with the on-site Coulomb correction $^{62}(\mathrm{DFTB}+\mathrm{U})$ are in quantitatively agreement with those by $\mathrm{DFT}+\mathrm{U}$ and hybrid functional methods. The excellent performance of DFTB on magnetite provides an alternative and cheap approach to the modelling of realistically large magnetite nanoparticles, which are the crucial systems in nanomedicine.

\section{METHODS}

The SCC-DFTB method is an approximated DFT-based method that derives from the second-order expansion of the KohnSham total energy in DFT with respect to the electron density fluctuations. The SCC-DFTB total energy can be defined as

$$
E_{\text {tot }}=\sum_{i}^{o c c} \varepsilon_{i}+\frac{1}{2} \sum_{\alpha, \beta}^{N} \gamma_{\alpha \beta} \Delta q_{\alpha} \Delta q_{\beta}+E_{r e p},
$$

where the first term is the sum of the one-electron energies $\varepsilon_{i}$ coming from the diagonalization of an approximated Hamiltonian matrix. $\Delta q_{\alpha}$ and $\Delta q_{\beta}$ are the induced charges on the atoms $\alpha$ and $\beta$, respectively, and $\gamma_{\alpha \beta}$ is a Coulombic-like interaction potential. $\mathrm{E}_{\text {rep }}$ is a short-range pairwise repulsive potential. More details about the SCC-DFTB method can be found in Refs. 45, 63, and 64. From now on, DFTB will be used as shorthand for SCC-DFTB.

All the DFTB calculations were performed by the DFTB + package. ${ }^{65}$ For the $\mathrm{Fe}-\mathrm{Fe}$ and $\mathrm{O}-\mathrm{O}$ interactions, we used the "trans3d-0-1" and "mio-1-1" set of parameters as reported previously. ${ }^{45,52}$ For the $\mathrm{Fe}-\mathrm{O}$ interactions, we first tried the SlaterKoster files from "trans3d-0-1." Then we refitted the repulsive part to improve its performance. To properly deal with the strong correlation effects among Fe 3 d electrons, DFTB $+\mathrm{U}^{62}$ with an effective $\mathrm{U}-\mathrm{J}$ value of $3.5 \mathrm{eV}$ was adopted according to our previous work on magnetite bulk and (001) surfaces based on DFT. ${ }^{34,44}$ The convergence criterion of $10^{-4}$ a.u. for force was used during geometry optimization, and the convergence threshold on the self-consistent charge (SCC) procedure was set to be $10^{-5}$ a.u.

For bulk magnetite, the conventional cell containing 32 oxygen atoms and 24 iron atoms was adopted and the $\mathrm{k}$ points generated by the Monkhorst-Pack scheme were chosen to be $6 \times 6 \times 6$. For the $\mathrm{Fe}_{3} \mathrm{O}_{4}(001)$ surface, two structural models were considered, DBT and SCV, as we used in our previous paper. ${ }^{34}$ An inversion symmetric slab with 17 atomic layers was adopted for both DBT and SCV structures (see Fig. 2). A $(\sqrt{ } 2 \times \sqrt{ } 2) \mathrm{R} 45^{\circ}$ supercell for the (001) surface was used for the DBT and SCV models containing $124\left(\mathrm{Fe}_{52} \mathrm{O}_{72}\right)$ and $122\left(\mathrm{Fe}_{50} \mathrm{O}_{72}\right)$ atoms, respectively. In the $\mathrm{z}$ direction, a vacuum of more than $12 \AA$ was introduced to avoid the spurious interaction between periodic images. Five atomic layers in the middle are kept fixed to the bulk position, and the other layers are fully relaxed during the geometry optimization. For the surfaces, the $\mathrm{K}$ point mesh was $6 \times 6 \times 1$.

For all the details on the DFT $+\mathrm{U}$ and HSE ${ }^{66}$ (Heyd-ScuseriaErnzerhof) calculations on magnetite bulk and surface systems, the reader is referred to our previous studies where they are reported. ${ }^{34,44}$

\section{RESULTS AND DISCUSSION}

The geometry of bulk magnetite was first fully relaxed with both DFTB and DFTB + U methods with the Slater-Koster files from "trans3d-0-1" and "mio-1-1" libraries, labeled DFTB-lib and DFTB + U-lib, respectively. The optimized lattice parameters by different methods together with the experimental value are listed in Table I. The lattice parameters given by DFTB-lib and DFTB + U-lib are much larger than the experimental value ${ }^{67}$ and values from Perdew Burke Ernzerhof (PBE), PBE + U, HSE, reported previously. ${ }^{44}$ The bad agreement is not surprising because the $\mathrm{Fe}-\mathrm{O}$ Slater-Koster files were fitted for bioinorganic systems. ${ }^{52}$

To improve the performance of DFTB on magnetite, we refitted the repulsive part of the Fe-O Slater-Koster file [the $E_{\text {rep }}$ term in formula (1)]. To do this, DFT calculations with the Perdew Burke Ernzerhof (PBE) functional were performed for the conventional cell of magnetite using the plane-wave-based Quantum ESPRESSO package. ${ }^{68}$ The detailed setup can be found in our previous paper for bulk 
TABLE I. Lattice parameter (a) for the conventional cell of bulk magnetite obtained by different methods.

\begin{tabular}{lcccccrrr}
\hline \hline Methods & DFTB-lib & DFTB + U-lib & DFTB-refitted & DFTB + U-refitted & PBE $^{44}$ & PBE + U & HSE ${ }^{44}$ & Expt. ${ }^{67}$ \\
\hline $\mathrm{a}(\AA)$ & 8.763 & 8.898 & 8.314 & 8.488 & 8.380 & 8.491 & 8.389 & 8.394 \\
\hline \hline
\end{tabular}

magnetite. ${ }^{44}$ A series of calculations for bulk magnetite with different lattice parameters were performed with both DFTB-lib and PBE to obtain the energy dependence on the $\mathrm{Fe}-\mathrm{O}$ distance. For each lattice parameter, the coordinates for all the atoms in the conventional cell were fully relaxed with PBE. The energy difference between electronic energy from DFTB-lib and binding energy from PBE can be viewed as the repulsive energy in formula (1). The $\mathrm{E}_{\text {rep }}$ is a pairwise repulsive potential. However, there are two kinds of $\mathrm{Fe}-\mathrm{O}$ bonds in bulk magnetite, $\mathrm{Fe}_{\mathrm{Oct}}-\mathrm{O}$ bond, and $\mathrm{Fe}_{\mathrm{Tet}}-\mathrm{O}$ bond. Therefore, to represent the repulsive energy as a function of $\mathrm{Fe}-\mathrm{O}$ distance, a special average $(\mathrm{R})$ of $\mathrm{Fe}_{\mathrm{Tet}}-\mathrm{O}$ and $\mathrm{Fe}_{\mathrm{Oct}}-\mathrm{O}$ distances $\left(\mathrm{R}_{\mathrm{Tet}}\right.$ and $\left.\mathrm{R}_{\mathrm{Oct}}\right)$ was computed as

$$
\mathrm{R}=\sqrt{\frac{4 R_{T e t}^{2} R_{O c t}^{2}}{R_{T e t}^{2}+3 R_{O c t}^{2}}} .
$$

The calculated $\mathrm{E}_{\text {rep }}$ per $\mathrm{Fe}-\mathrm{O}$ pair as a function of the average $\mathrm{Fe}-\mathrm{O}$ distance is shown in Fig. 1 (black points). This was fitted by a polynomial $^{63}$

$$
E_{\text {rep }}(R)= \begin{cases}\sum_{n=2} d_{n}\left(R_{c}-R\right)^{n}, & \text { for } R<R_{c} \\ 0, & \text { otherwise, }\end{cases}
$$

where $R_{c}$ is the cutoff radius and $d_{n}$ are the coefficients. The best fitting is given by $\mathrm{R}_{c}=4.3$ Bohrs. The fitted $\mathrm{E}_{\text {rep }}$ as a function of the average $\mathrm{Fe}-\mathrm{O}$ distance are plotted in Fig. 1 (red line).

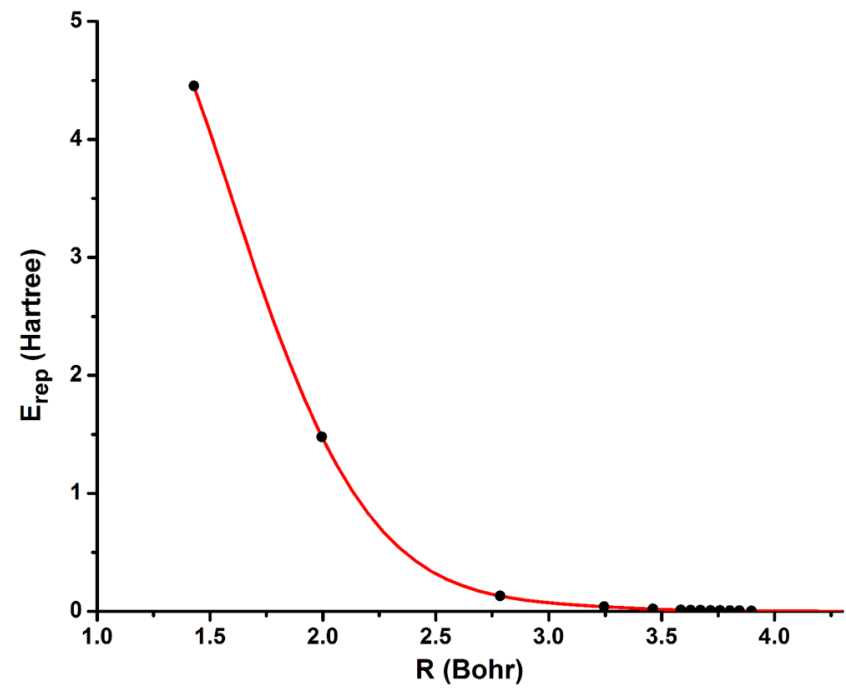

FIG. 1. The repulsive energy as a function of the average Fe-O distance in bulk magnetite. The black points are obtained by calculations, and the red line is the plotting of the fitted $E_{\text {rep }}$
We used the refitted $\mathrm{Fe}-\mathrm{O}$ Slater-Koster file to fully relax the geometry of bulk magnetite again with both DFTB and DFTB + U methods, labeled DFTB-refitted and DFTB + U-refitted, respectively. DFTB + U-refitted results in a lattice parameter similar to that from $\mathrm{PBE}+\mathrm{U}$ and HSE, only about $1.1 \%$ larger than the experimental value. ${ }^{67}$ In contrast, DFTB-refitted underestimates the lattice parameter with respect to the experimental value, similarly to the PBE method. Therefore, DFTB + U with the refitted Fe-O SlaterKoster file can well describe the structural properties of bulk magnetite and will be adopted hereafter. From now on, we will refer to $\mathrm{DFTB}+\mathrm{U}$-refitted as DFTB + U.

We now discuss how the DFTB $+\mathrm{U}$ method describes the electronic properties. The distribution of $\mathrm{Fe}^{2+}$ and $\mathrm{Fe}^{3+}$ at different lattice sites is shown in Table II. All the Fe ions at tetrahedral sites are $3+$, in accordance with PBE $+\mathrm{U}$ and HSE. ${ }^{44}$ However, DFTB + U cannot reproduce the charge disproportionation for the bulk magnetite, i.e., all the Fe ions at octahedral sites have the same valence states, which can be viewed as $\mathrm{Fe}^{2.5+}$. This is because the high structural symmetry of bulk magnetite prevents an approximated DFTbased method to correctly describe the symmetry breaking of the wavefunction that is required to distinguish $\mathrm{Fe}^{3+}$ and $\mathrm{Fe}^{2+}$ at geometrically equivalent octahedral sites. Such a delicate effect can be observed at the DFT $+\mathrm{U}$ and hybrid functional level of theory. ${ }^{44}$ All the Fe ions at tetrahedral sites couple antiferromagnetically with Fe ions at octahedral sites (see Table II). DFTB + U slightly overestimates the atomic magnetic moment compared with $\mathrm{PBE}+\mathrm{U}$ and HSE. Note that the total magnetic moment for the conventional cell is fixed to be $32 \mu_{\mathrm{B}}$ for DFTB $+\mathrm{U}$ calculations according to the value given by $\mathrm{PBE}+\mathrm{U}$.

The projected density of states (PDOS) on the $d$ states of different Fe ions is calculated by DFTB $+\mathrm{U}$ [Fig. 2(a)]. The PDOS shows a half-metal characteristic with some spin down states

TABLE II. Number of Fe ions at tetrahedral and octahedral sites with different valences $\left(\mathrm{N}_{\mathrm{Tet}}{ }^{3+}, \mathrm{N}_{\mathrm{Oct}}{ }^{3+}\right.$, and $\left.\mathrm{N}_{\mathrm{Oct}}{ }^{2+}\right)$, charge difference between $\mathrm{Fe}_{\mathrm{Oct}}{ }^{3+}$ and $\mathrm{Fe}_{\mathrm{Oct}}{ }^{2+}$ $(\Delta \mathrm{Q})$, magnetic moment of $\mathrm{Fe}$ ions at tetrahedral and octahedral sites $\left(\mathrm{m}_{\mathrm{Tet}}, \mathrm{m}_{\mathrm{Oct}}{ }^{3+}\right.$, and $\left.\mathrm{m}_{\mathrm{Oct}}{ }^{2+}\right)$ in bulk magnetite derived from different methods: DFTB $+\mathrm{U}, \mathrm{PBE}+\mathrm{U}$, and HSE.

\begin{tabular}{lccc}
\hline \hline & DFTB $+\mathrm{U}$ & $\mathrm{PBE}+\mathrm{U}^{44}$ & $\mathrm{HSE}^{44}$ \\
\hline $\mathrm{N}_{\mathrm{Tet}}{ }^{3+}$ & 8 & 8 & 8 \\
$\mathrm{~N}_{\text {Oct }}{ }^{3+}$ & 16 & 8 & 8 \\
$\mathrm{~N}_{\text {Oct }}{ }^{2+}$ & 0.0 & 8 & 8 \\
$\Delta \mathrm{Q}(\mathrm{e})$ & -4.53 & 0.27 & 0.32 \\
$\mathrm{~m}_{\text {Tet }}\left(\mu_{\mathrm{B}}\right)$ & 4.11 & 4.06 & -4.21 \\
$\mathrm{~m}_{\text {Oct }}{ }^{3+}\left(\mu_{\mathrm{B}}\right)$ & & 3.62 & 4.27 \\
$\mathrm{~m}_{\text {Oct }}{ }^{2+}\left(\mu_{\mathrm{B}}\right)$ & & & 3.79 \\
\hline \hline
\end{tabular}


(a) DFTB+U

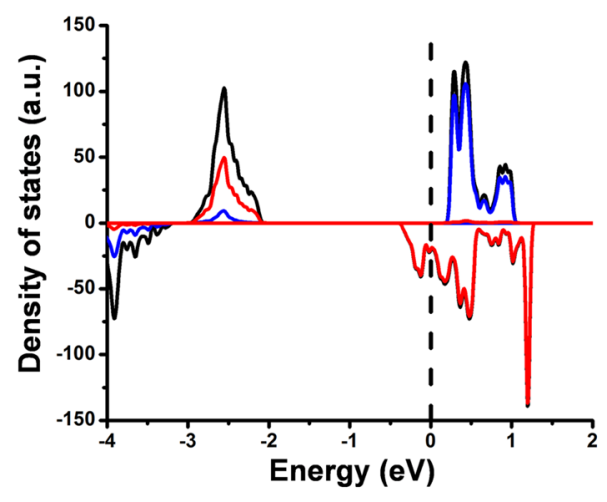

(b) $\mathrm{PBE}+\mathrm{U}$

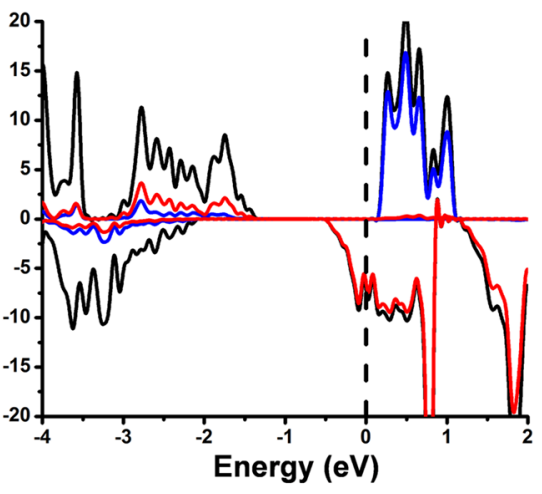

(c) HSE

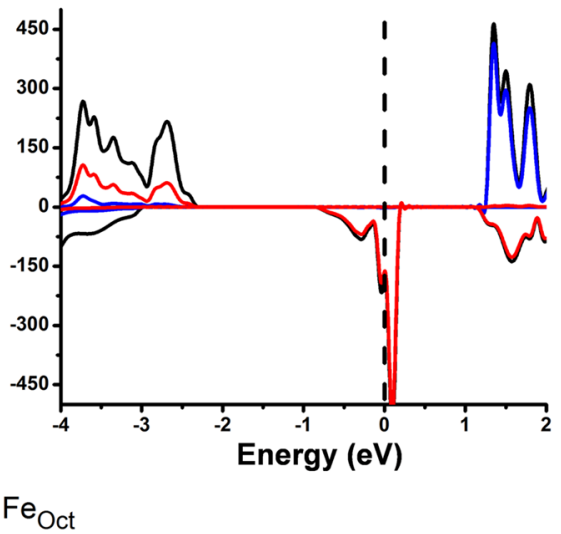

FIG. 2. Projected density of states on the d states of different Fe ions in bulk magnetite calculated with (a) DFTB $+U$, (b) PBE $+U$, and (c) HSE. Legend of colors is on the bottom. Data for (b) and (c) are taken from our previous paper. ${ }^{44}$

from Feoct at the Fermi level. This agrees well with those obtained by PBE + U [(Fig. 2(b)] and HSE [Fig. 2(c)] with the symmetry constrained wavefunction. ${ }^{44}$ The lack of bandgap for the spin down channel is because of the uniform valence state of Fe ions $\left(\mathrm{Fe}^{2.5+}\right)$ at octahedral sites, as discussed previously. ${ }^{44} \mathrm{~A}$ bandgap between $d$ states of $\mathrm{Fe}$ at octahedral and tetrahedral sites exists for the spin up channel, which is similar to the results from $P B E+U$ and HSE.
To check the performance of DFTB $+\mathrm{U}$ on $\mathrm{Fe}_{3} \mathrm{O}_{4}$ surfaces, the two proposed models (DBT and SCV, as discussed in the Introduction) of the well-studied (001) surface were fully relaxed with DFTB + U. For the DBT surface, no undulation along the surface $\mathrm{Fe}_{\mathrm{Oct}}$ rows was observed [Fig. 3(a)]. In contrast, the undulations in the SCV surface model are very pronounced [Fig. 3(b)]. These observations agree well with previous results based on DFT $+U$ and HSE calculations. ${ }^{20,34}$ For both DBT and SCV surfaces, the distance (a) DBT
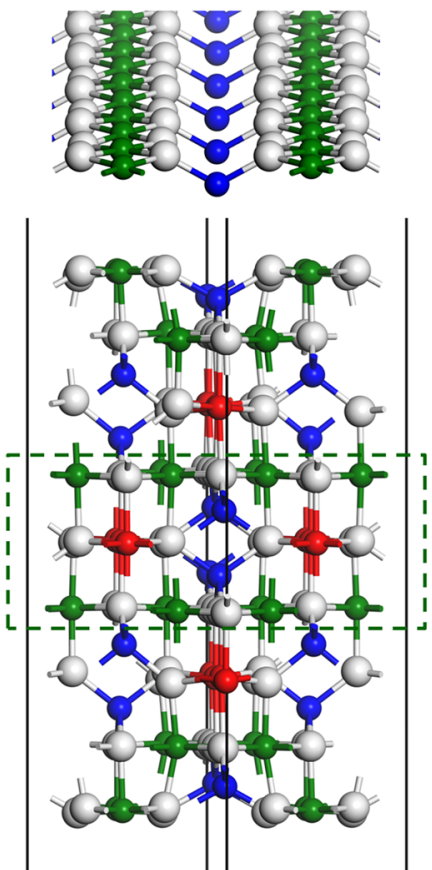

- $\mathrm{Fe}_{\mathrm{Tet}}{ }^{3+}$ (b) SCV
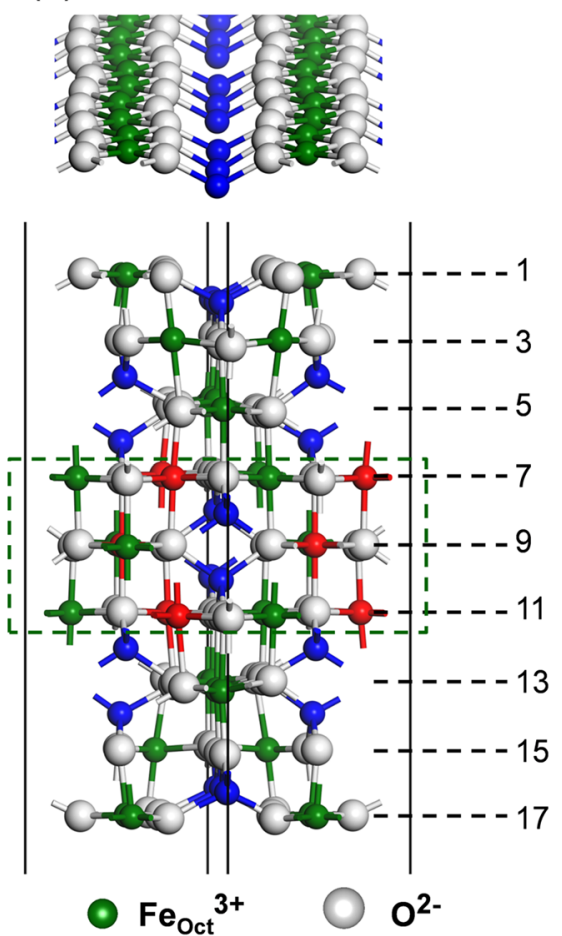

FIG. 3. Top and side views of optimized atomic structures of the (a) DBT and (b) $\mathrm{SCV} \mathrm{Fe}_{3} \mathrm{O}_{4}(001)$ surface models by $\mathrm{DFTB}+\mathrm{U}$. Layers are numbered on the right. Atomic layers in the dashed rectangles are kept fixed in the bulk positions during atomic relaxation. 
TABLE III. Numbers of Fe ions with different valences at different sites $\left(\mathrm{N}_{\mathrm{Tet}}{ }^{3+}, \mathrm{N}_{\mathrm{Oct}}{ }^{3+}\right.$, and $\left.\mathrm{N}_{\mathrm{Oct}}{ }^{2+}\right)$, charge difference between $\mathrm{Fe}_{\mathrm{Oct}}{ }^{3+}$ and $\mathrm{Fe}_{\mathrm{Oct}}{ }^{2+}(\Delta \mathrm{Q})$, magnetic moment of Fe ions at tetrahedral and octahedral sites $\left(\mathrm{m}_{\mathrm{Tet}}, \mathrm{m}_{\mathrm{Oct}}{ }^{3+}\right.$, and $\left.\mathrm{m}_{\mathrm{Oct}}{ }^{2+}\right)$ in $\mathrm{Fe}_{3} \mathrm{O}_{4}(001)$ surfaces derived from different methods: DFTB $+\mathrm{U}, \mathrm{PBE}+\mathrm{U}$, and $\mathrm{HSE}$.

\begin{tabular}{|c|c|c|c|c|c|c|}
\hline & \multicolumn{3}{|c|}{ DBT } & \multicolumn{3}{|c|}{ SCV } \\
\hline & $\mathrm{DFTB}+\mathrm{U}$ & $\mathrm{PBE}+\mathrm{U}^{34}$ & $\mathrm{HSE}^{34}$ & $\mathrm{DFTB}+\mathrm{U}$ & $\mathrm{PBE}+\mathrm{U}^{34}$ & $\mathrm{HSE}^{34}$ \\
\hline $\mathrm{N}_{\mathrm{Tet}}{ }^{3+}$ & 16 & 16 & 16 & 18 & 18 & 18 \\
\hline $\mathrm{N}_{\text {Oct }}^{3+}$ & 24 & 24 & 24 & 26 & 26 & 26 \\
\hline $\mathrm{N}_{\mathrm{Oct}}{ }^{2+}$ & 12 & 12 & 12 & 6 & 6 & 6 \\
\hline$\Delta \mathrm{Q}(\mathrm{e})$ & 0.63 & 0.35 & 0.37 & 0.65 & 0.34 & 0.38 \\
\hline $\mathrm{m}_{\mathrm{Tet}}\left(\mu_{\mathrm{B}}\right)$ & -4.51 to -4.57 & -3.96 to -3.98 & -4.20 to -4.24 & -4.53 to -4.58 & -3.92 to -3.99 & -4.18 to -4.24 \\
\hline $\mathrm{m}_{\mathrm{Oct}}^{3+}\left(\mu_{\mathrm{B}}\right)$ & $4.50-4.61$ & $4.05-4.09$ & $4.29-4.30$ & $4.49-4.59$ & $4.04-4.09$ & $4.26-4.33$ \\
\hline $\mathrm{m}_{\mathrm{Oct}}^{2+}\left(\mu_{\mathrm{B}}\right)$ & $3.71-3.76$ & $3.62-3.67$ & $3.75-3.84$ & $3.70-3.74$ & $3.60-3.66$ & $3.76-3.83$ \\
\hline
\end{tabular}

(a) DBT DFTB+U

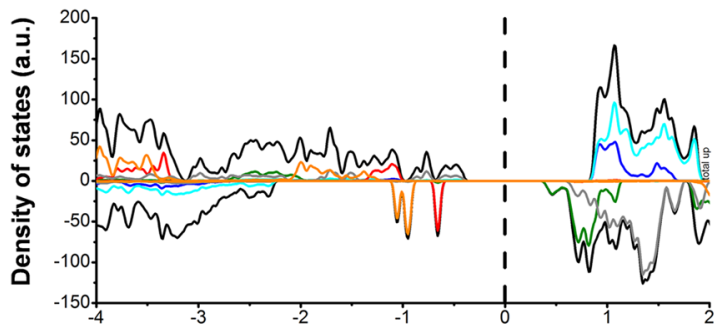

(c) DBT PBE+U

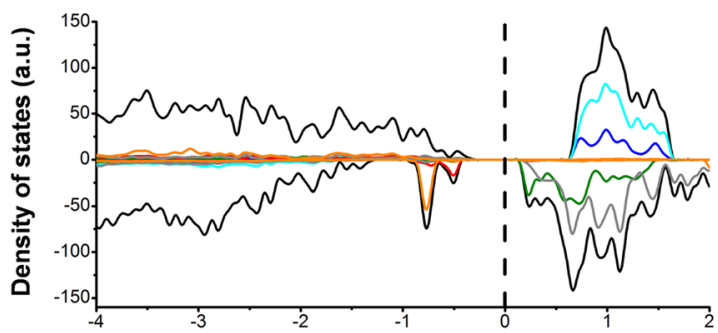

(e) DBT HSE

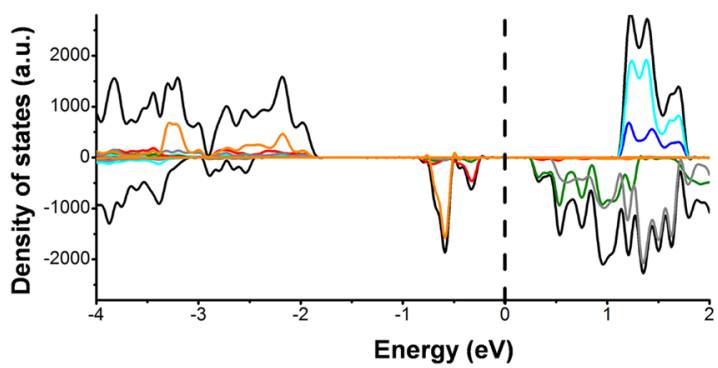

(b) SCV DFTB+U

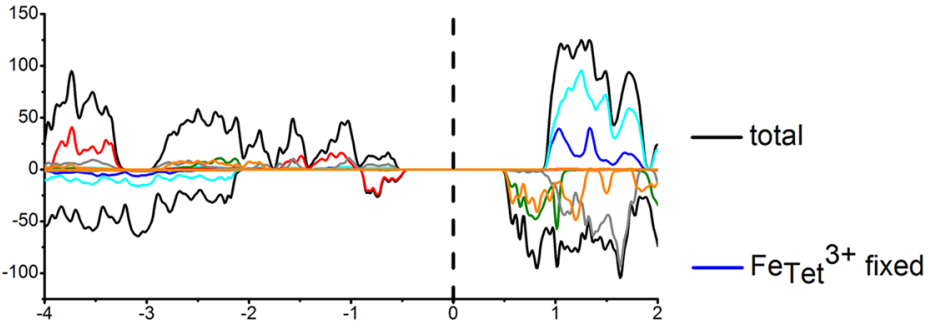

(d) SCV PBE+U

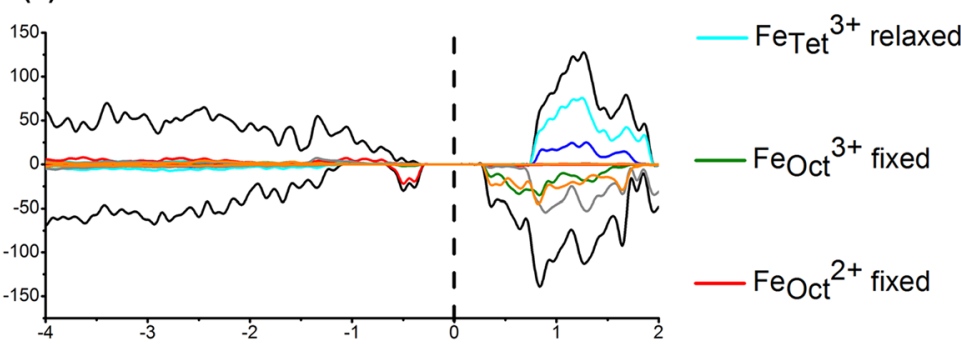

(f) SCV HSE

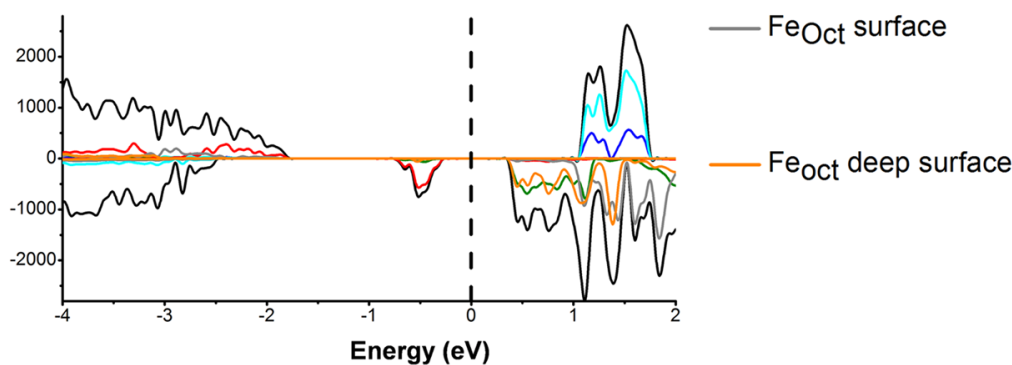

FIG. 4. PDOS on the $d$ states of different Fe ions in the SCV and DBT slab models. (a) and (b) are calculated using DFTB + U; (c) and (d) are calculated using PBE + U; ${ }^{34}$ and $(e)$ and (f) are calculated using HSE. ${ }^{34}$ The legend of colors is on the right. The black lines represent the total DOS. The blue and cyan lines represent PDOS on the $d$ states of $\mathrm{Fe}_{\mathrm{Tet}^{3}}{ }^{3+}$ in the fixed layers and relaxed layers, respectively. The green and red lines represent PDOS on the $d$ states of $\mathrm{Fe}_{\mathrm{Oct}^{3+}}{ }^{3+} \mathrm{and} \mathrm{Fe}_{\mathrm{Oct}^{2+}}{ }^{2}$ in the fixed layers, respectively. The gray lines represent PDOS on the $d$ states of Fe oct $_{\text {in }}$ the surface layers (layer $1+$ layer $3+$ layer15 + layer17). The orange lines represent PDOS on the $d$ states of $\mathrm{Fe}_{\mathrm{Oct}}$ in the deep surface layers (layer5 + layer13). The Fermi level is scaled to zero as indicated by the dashed black lines. 
between the first two layers is largely compressed with respect to the bulk value ( 0.828 and $0.812 \AA$ vs. $1.061 \AA$, respectively), while the distance between the second and the third layers is only slightly changed. The relaxation in the surface layers is also in accordance with that obtained by DFT $+\mathrm{U}$ and HSE methods. ${ }^{34}$ Therefore, $\mathrm{DFTB}+\mathrm{U}$ can well describe the structural details of magnetite surfaces.

Fe ions with different valences at tetrahedral and octahedral sites are marked with different colors and shown in Fig. 3. The $\mathrm{DFTB}+\mathrm{U}$ method is capable of reproducing the charge disproportionation of $\mathrm{Fe}$ ions at the octahedral sites. The surface cut to build the slab models reduces the symmetry with respect to the bulk and makes it easier for the DFTB + U method to break the wavefunction symmetry and thus distinguish $\mathrm{Fe}^{2+}$ and $\mathrm{Fe}^{3+}$ at different sites. In the outmost two layers of the DBT model, all Feoct are $\mathrm{Fe}^{3+}$ due to the low Fe:O stoichiometry in surface layers. On the contrary, all Feoct in layers 5 and 13 (hereafter called as "deep surface layers") are $\mathrm{Fe}^{2+}$. In contrast, all $\mathrm{Fe}_{\mathrm{Oct}}$ in the fully relaxed layers are $\mathrm{Fe}^{3+}$ for the SCV model [Fig. 3(b)] as a consequence of even lower $\mathrm{Fe}: \mathrm{O}$ ratio in the surface layers due to the $\mathrm{Fe}$ vacancies present in the third layer. The high $\mathrm{Fe}^{3+}$ density at surface layers is consistent with XPS measurements. ${ }^{20}$ For both DBT and SCV surfaces, in those layers that are kept fixed during atomic relaxation (from 7 th layer to 11th layer), $\mathrm{Fe}^{2+}$ and $\mathrm{Fe}^{3+}$ ions alternate, as observed in the bulk phase. ${ }^{44}$ Iron cations at tetrahedral sites are always $\mathrm{Fe}^{3+}$, whether at the surface or in the pseudo-bulk layers. The distribution of $\mathrm{Fe}^{2+}$ and $\mathrm{Fe}^{3+}$ in the surface slabs just described for DFTB $+\mathrm{U}$ calculations agrees well with that obtained by PBE $+\mathrm{U}$ and HSE. ${ }^{34}$ In addition, the numbers of $\mathrm{Fe}$ ions with different valences at different sites are exactly the same as those obtained by PBE $+\mathrm{U}$ and HSE (listed in Table III). Therefore, DFTB + U can quantitatively reproduce the results from high level DFT calculations (PBE $+\mathrm{U}$ and HSE). The charge difference between $\mathrm{Fe}_{\mathrm{Oct}}{ }^{3+}$ and $\mathrm{Fe}_{\mathrm{Oct}}{ }^{2+}$ obtained by DFTB + $\mathrm{U}$ is around $0.65 \mathrm{e}$, which is larger than that obtained from $\mathrm{PBE}+$ $\mathrm{U}$ and HSE (around $0.35 \mathrm{e}$ ). As shown in Table III, the atomic magnetic moment of $\mathrm{Fe}^{2+}$ from DFTB $+\mathrm{U}$ agrees well with that by $\mathrm{PBE}+$ $\mathrm{U}$ and HSE. However, the magnetic moment of $\mathrm{Fe}^{3+}$ is slightly overestimated especially compared with that from PBE + U calculations. This is consistent with the larger charge difference $\Delta \mathrm{Q}$ obtained by $\mathrm{DFTB}+\mathrm{U}$. Note that for DFTB + U calculations, the total magnetic moments for the DBT and SCV surfaces have been fixed to $88 \mu_{\mathrm{B}}$ and $64 \mu_{\mathrm{B}}$, according to the values obtained with the PBE $+\mathrm{U}$ method. ${ }^{34}$

The PDOS on the $d$ states of different Fe ions for both SCV and DBT surface models is calculated by DFTB $+U$ and shown in Figs. 4(a) and 4(b). PDOS calculated by PBE + U and HSE from a previous work by some of $\mathrm{us}^{34}$ is also plotted here [Figs. 4(c)-4(f)] for comparison. In general, the PDOS from DFTB + U agrees well with that from PBE $+\mathrm{U}$ and HSE. Both DBT and SCV surfaces are calculated to be semiconductors with a bandgap between the $\mathrm{t}_{2 \mathrm{~g}}$ states from $\mathrm{Fe}_{\mathrm{Oct}}{ }^{2+}$ and $\mathrm{Fe}_{\mathrm{Oct}}{ }^{3+}$ in the inner bulk-like layers. The bandgap values by DFTB $+\mathrm{U}$ are $0.89 \mathrm{eV}$ and $0.85 \mathrm{eV}$ for DBT and SCV surfaces, respectively, which are slightly larger than those by $\mathrm{PBE}+\mathrm{U}(0.60 \mathrm{eV}$ and $0.61 \mathrm{eV}$, respectively) and by HSE $(0.56 \mathrm{eV}$ and $0.69 \mathrm{eV}$, respectively). The characteristic peak in spin down states at about $-0.5 \mathrm{eV}$ observed both in experimental photoemission spectroscopy ${ }^{69-71}$ and in previous computational studies ${ }^{34}$ is also well reproduced. Considering the valence bands of the PDOS, DFTB + U is closer to $\mathrm{PBE}+\mathrm{U}$ than to HSE. Based on the results just discussed above, we may conclude that DFTB + U can also rather satisfactorily describe not only the structural but also the electronic properties of magnetite surfaces.

\section{CONCLUSION}

In summary, in this work we have proposed a new DFTB parametrization of the $\mathrm{Fe}-\mathrm{O}$ interactions based on previous SlaterKoster files, which enables it to describe rather accurately magnetite bulk and surface systems. The performance of DFTB $+U$ with the proposed parameters is well assessed by investigating both magnetite bulk and (001) surfaces and through comparison with available experimental data and previous results by more sophisticated DFT methods (DFT $+\mathrm{U}$ and hybrid functional HSE). ${ }^{34,44}$

We observe that the lattice parameter of bulk magnetite can be calculated precisely by DFTB $+\mathrm{U}$ with an error of only $1.1 \%$ compared with the experimental value. The $\mathrm{Fe}^{2+} / \mathrm{Fe}^{3+}$ charge disproportionation in bulk magnetite cannot be reproduced due to the high symmetry. Nevertheless, the electronic properties of bulk magnetite obtained by DFTB $+\mathrm{U}$ agree well with those obtained by DFT $+\mathrm{U}$ and HSE with the symmetry constrained wavefunction.

For the (001) surface, both the structural and electronic properties obtained by DFTB $+\mathrm{U}$ are in quantitative agreement with those obtained by DFT $+\mathrm{U}$ and HSE, including the charge disproportionation and charge distribution.

The excellent performance of DFTB $+\mathrm{U}$ on magnetite bulk and surfaces, in comparison to more sophisticated techniques, provides an efficient compromise between computational cost and accuracy for their simulation and, more interestingly, for the simulation of realistically large magnetite nanostructures. For example, with $\mathrm{DFTB}+\mathrm{U}$ we could perform the full atomic relaxation of a magnetite nanocube containing about 1500 atoms with the edge length of $2.3 \mathrm{~nm}$. Therefore, the DFTB $+\mathrm{U}$ approach can handle magnetite nanoparticles of realistic size, whose simulation would be prohibitive with HSE and PBE + U methods. This work paves the way to innovative quantum mechanical studies on magnetite based nanomaterials, which attract increasing attention in medical applications.

\section{ACKNOWLEDGMENTS}

The authors are grateful to Lorenzo Ferraro for his technical help. The project has received funding from the European Research Council (ERC) under the European Union's HORIZON2020 research and innovation programme (ERC Grant Agreement No. 647020).

\section{REFERENCES}

${ }^{1}$ W. Wei, W. Zhaohui, Y. Taekyung, J. Changzhong, and K. Woo-Sik, Sci. Technol. Adv. Mater. 16, 023501 (2015).

${ }^{2}$ A. G. Roca, R. Costo, A. F. Rebolledo, S. Veintemillas-Verdaguer, P. Tartaj, T. González-Carreño, M. P. Morales, and C. J. Serna, J. Phys. D: Appl. Phys. 42, 224001 (2009).

${ }^{3}$ A. K. Gupta and M. Gupta, Biomaterials 26, 3995 (2005).

${ }^{4}$ C. Sun, J. S. Lee, and M. Zhang, Adv. Drug Delivery Rev. 60, 1252 (2008).

${ }^{5}$ Q. A. Pankhurst, J. Connolly, S. K. Jones, and J. Dobson, J. Phys. D: Appl. Phys. 36, R167 (2003). 
${ }^{6}$ S. Laurent, D. Forge, M. Port, A. Roch, C. Robic, L. V. Elst, and R. N. Muller, Chem. Rev. 108, 2064 (2008).

${ }^{7}$ M. Colombo, S. Carregal-Romero, M. F. Casula, L. Gutiérrez, M. P. Morales, I. B. Böhm, J. T. Heverhagen, D. Prosperi, and W. J. Parak, Chem. Soc. Rev. 41, 4306 (2012).

${ }^{8}$ E. J. W. Verwey and J. H. de Boer, Recl. Trav. Chim. Pays-Bas 55, 531 (1936).

${ }^{9}$ G. S. Parkinson, Surf. Sci. Rep. 71, 272 (2016).

${ }^{10}$ J. M. Gaines, P. J. H. Bloemen, J. T. Kohlhepp, C. W. T. Bulle-Lieuwma, R. M. Wolf, A. Reinders, R. M. Jungblut, P. A. A. van der Heijden, J. T. W. M. van Eemeren, J. aan de Stegge, and W. J. M. de Jonge, Surf. Sci. 373, 85 (1997).

${ }^{11}$ G. Tarrach, D. Bürgler, T. Schaub, R. Wiesendanger, and H. J. Güntherodt, Surf. Sci. 285, 1 (1993).

${ }^{12}$ S. A. Chambers, S. Thevuthasan, and S. A. Joyce, Surf. Sci. 450, L273 (2000).

${ }^{13}$ A. V. Mijiritskii and D. O. Boerma, Surf. Sci. 486, 73 (2001).

${ }^{14}$ B. Stanka, W. Hebenstreit, U. Diebold, and S. A. Chambers, Surf. Sci. 448, 49 (2000).

${ }^{15}$ F. C. Voogt, T. Fujii, P. J. M. Smulders, L. Niesen, M. A. James, and T. Hibma, Phys. Rev. B 60, 11193 (1999).

${ }^{16}$ R. Pentcheva, F. Wendler, H. L. Meyerheim, W. Moritz, N. Jedrecy, and M. Scheffler, Phys. Rev. Lett. 94, 126101 (2005).

${ }^{17}$ Z. Łodziana, Phys. Rev. Lett. 99, 206402 (2007).

${ }^{18}$ R. Pentcheva, W. Moritz, J. Rundgren, S. Frank, D. Schrupp, and M. Scheffler, Surf. Sci. 602, 1299 (2008).

${ }^{19}$ Z. Novotný, G. Argentero, Z. Wang, M. Schmid, U. Diebold, and G. S. Parkinson, Phys. Rev. Lett. 108, 216103 (2012).

${ }^{20}$ R. Bliem, E. McDermott, P. Ferstl, M. Setvin, O. Gamba, J. Pavelec, M. A. Schneider, M. Schmid, U. Diebold, P. Blaha, L. Hammer, and G. S. Parkinson, Science 346, 1215 (2014).

${ }^{21}$ B. Arndt, R. Bliem, O. Gamba, J. E. S. van der Hoeven, H. Noei, U. Diebold, G. S. Parkinson, and A. Stierle, Surf. Sci. 653, 76 (2016).

${ }^{22}$ R. G. S. Sofin, S. K. Arora, and I. V. Shvets, Phys. Rev. B 83, 134436 (2011).

${ }^{23}$ R. Jansen, V. A. M. Brabers, and H. van Kempen, Surf. Sci. 328, 237 (1995).

${ }^{24}$ G. Maris, O. Shklyarevskii, L. Jdira, J. G. H. Hermsen, and S. Speller, Surf. Sci. 600, 5084 (2006).

${ }^{25}$ Y. Li, K. Yao, and Z. Liu, Surf. Sci. 601, 876 (2007).

${ }^{26}$ Y. Li, K. Yao, and Z. Liu, Front. Phys. China 2, 76 (2007).

${ }^{27}$ X. Yu, Y. Li, Y. Li, J. Wang, and H. Jiao, J. Phys. Chem. C 117, 7648 (2013).

${ }^{28}$ G. S. Parkinson, Z. Novotný, P. Jacobson, M. Schmid, and U. Diebold, J. Am. Chem. Soc. 133, 12650 (2011).

${ }^{29}$ T. Kendelewicz, P. Liu, C. S. Doyle, G. E. Brown, E. J. Nelson, and S. A. Chambers, Surf. Sci. 453, 32 (2000).

${ }^{30}$ T. Kendelewicz, S. Kaya, J. T. Newberg, H. Bluhm, N. Mulakaluri, W. Moritz, M. Scheffler, A. Nilsson, R. Pentcheva, and G. E. Brown, J. Phys. Chem. C 117, 2719 (2013).

${ }^{31}$ N. Mulakaluri, R. Pentcheva, M. Wieland, W. Moritz, and M. Scheffler, Phys. Rev. Lett. 103, 176102 (2009).

${ }^{32}$ S. Liu, S. Wang, W. Li, J. Guo, and Q. Guo, J. Phys. Chem. C 117, 14070 (2013). ${ }^{33}$ N. Mulakaluri, R. Pentcheva, and M. Scheffler, J. Phys. Chem. C 114, 11148 (2010).

${ }^{34}$ H. Liu and C. Di Valentin, Nanoscale 10, 11021 (2018).

${ }^{35}$ M. Meiera, J. Hulva, Z. Jakub, J. Pavelec, M. Setvin, R. Bliem, M. Schmid, U. Diebold, C. Franchini, and G. S. Parkinson, Proc. Natl. Acad. Sci. U. S. A. 115, E5642 (2018).

${ }^{36}$ U. Aschauer and A. Selloni, J. Chem. Phys. 143, 044705 (2015).

${ }^{37}$ P. Xue, Z. Fu, X. Chu, Y. Zhang, and Z. Yang, Appl. Surf. Sci. 317, 752 (2014).

${ }^{38}$ O. Gamba, H. Noei, J. Pavelec, R. Bliem, M. Schmid, U. Diebold, A. Stierle, and G. S. Parkinson, J. Phys. Chem. C 119, 20459 (2015).

${ }^{39}$ Z. Li, D. V. Potapenko, K. Taeg Rim, M. Flytzani-Stephanopoulos, G. W. Flynn, R. M. Osgood, X. Wen, and E. R. Batista, J. Phys. Chem. C 119, 1113 (2015).
${ }^{40}$ R. A. Harris, H. van der Walt, and P. M. Shumbula, J. Mol. Struct. 1048, 18 (2013).

${ }^{41}$ R. A. Harris, P. M. Shumbula, and H. van der Walt, Langmuir 31, 3934 (2015).

${ }^{42}$ M. Patitsa, K. Karathanou, Z. Kanaki, L. Tzioga, N. Pippa, C. Demetzos, D. A. Verganelakis, Z. Cournia, and A. Klinakis, Sci. Rep. 7, 775 (2017).

${ }^{43}$ A. Hosseini nasr, H. Akbarzadeh, and R. Tayebee, J. Mol. Liq. 254, 64 (2018).

${ }^{44}$ H. Liu and C. Di Valentin, J. Phys. Chem. C 121, 25736 (2017).

${ }^{45}$ M. Elstner, D. Porezag, G. Jungnickel, J. Elsner, M. Haugk, Th. Frauenheim, S. Suhai, and G. Seifert, Phys. Rev. B 58, 7260 (1998).

${ }^{46}$ S. Markov, B. Aradi, C. Yam, H. Xie, T. Frauenheim, and G. Chen, IEEE Trans. Electron Devices 62, 696 (2015).

${ }^{47}$ A. Fihey, C. Hettich, J. Touzeau, F. Maurel, A. Perrier, C. Köhler, B. Aradi, and T. Frauenheim, J. Comput. Chem. 36, 2075 (2015).

${ }^{48}$ B. Szücs, Z. Hajnal, R. Scholz, S. Sanna, and T. Frauenheim, Appl. Surf. Sci. 234, 173 (2004).

${ }^{49}$ G. Seifert, H. Terrones, M. Terrones, G. Jungnickel, and T. Frauenheim, Phys. Rev. Lett. 85, 146 (2000).

${ }^{50}$ M. P. Lourenço, C. de Oliveira, A. F. Oliveira, L. Guimarães, and H. A. Duarte, J. Phys. Chem. C 116, 9405 (2012).

${ }^{51}$ M. Bar-Sadan, A. N. Enyashin, S. Gemming, R. Popovitz-Biro, S. Y. Hong, Y. Prior, R. Tenne, and G. Seifert, J. Phys. Chem. B 110, 25399 (2006).

${ }^{52}$ G. Zheng, H. A. Witek, P. Bobadova-Parvanova, S. Irle, D. G. Musaev, R. Prabhakar, K. Morokuma, M. Lundberg, M. Elstner, C. Köhler, and T. Frauenheim, J. Chem. Theory Comput. 3, 1349 (2007).

${ }^{53}$ G. Dolgonos, B. Aradi, N. H. Moreira, and T. Frauenheim, J. Chem. Theory Comput. 6, 266 (2010).

${ }^{54}$ R. Luschtinetz, J. Frenzel, T. Milek, and G. Seifert, J. Phys. Chem. C 113, 5730 (2009).

${ }^{55}$ H. Fox, K. E. Newman, W. F. Schneider, and S. A. Corcelli, J. Chem. Theory Comput. 6, 499 (2010).

${ }^{56}$ V. C. Fuertes, C. F. A. Negre, M. B. Oviedo, F. P. Bonafé, F. Y. Oliva, and C. G. Sánchez, J. Phys.: Condens. Matter 25, 115304 (2013).

${ }^{57}$ S. Manzhos, G. Giorgi, and K. Yamashita, Molecules 20, 3371 (2015).

${ }^{58}$ G. A. Dolgonos, Surf. Sci. 621, 51 (2014).

${ }^{59}$ W. Li, K. Kotsis, and S. Manzhos, Phys. Chem. Chem. Phys. 18, 19902 (2016).

${ }^{60}$ S. Manzhos, Chem. Phys. Lett. 643, 16 (2016).

${ }^{61}$ D. Selli, G. Fazio, and C. Di Valentin, J. Chem. Phys. 147, 164701 (2017).

${ }^{62}$ B. Hourahine, S. Sanna, B. Aradi, C. Köhler, Th. Niehaus, and Th. Frauenheim, J. Phys. Chem. A 111, 5671 (2007).

${ }^{63}$ G. Seifert and J.-O. Joswig, Wiley Interdiscip. Rev.: Comput. Mol. Sci. 2, 456 (2012).

${ }^{64}$ M. Elstner and G. Seifert, Philos. Trans. R. Soc., A 372, 20120483 (2014).

${ }^{65}$ B. Aradi, B. Hourahine, and T. Frauenheim, J. Phys. Chem. A 111, 5678 (2007).

${ }^{66}$ J. Heyd and G. E. Scuseria, J. Chem. Phys. 118, 8207 (2003).

${ }^{67}$ J. P. Wright, J. P. Attfield, and P. G. Radaelli, Phys. Rev. B: Condens. Matter Mater. Phys. 66, 214422 (2002).

${ }^{68}$ P. Giannozzi, S. Baroni, N. Bonini, M. Calandra, R. Car, C. Cavazzoni, D. Ceresoli, G. L. Chiarotti, M. Cococcioni, I. Dabo, A. Dal Corso, S. de Gironcoli, S. Fabris, G. Fratesi, R. Gebauer, U. Gerstmann, C. Gougoussis, A. Kokalj, M. Lazzeri, L. Martin-Samos, N. Marzari, F. Mauri, R. Mazzarello, S. Paolini, A. Pasquarello, L. Paulatto, C. Sbraccia, S. Scandolo, G. Sclauzero, A. P Seitsonen, A. Smogunov, P. Umari, and R. M Wentzcovitch, J. Phys.: Condens. Matter 21, 395502 (2009).

${ }^{69}$ D. Schrupp, M. Sing, M. Tsunekawa, H. Fujiwara, S. Kasai, A. Sekiyama, S. Suga, T. Muro, V. A. M. Brabers, and R. Claessen, Europhys. Lett. 70, 789 (2005).

${ }^{70}$ J. G. Tobin, S. A. Morton, S. W. Yu, G. D. Waddill, I. K. Schuller, and S. A. Chambers, J. Phys.: Condens. Matter 19, 315218 (2007).

${ }^{71}$ J. H. Park, L. H. Tjeng, J. W. Allen, P. Metcalf, and C. T. Chen, Phys. Rev. B: Condens. Matter Mater. Phys. 55, 12813 (1997). 